\title{
Rhabdomyomatous Mesenchymal Hamartoma: A New Proposed Clinical Classification of Adult Onset Acquired Type
}

Yuko EHARA ${ }^{1}$, Yuichi YOSHIDA ${ }^{1}$, Shinji KATAOKA ${ }^{2}$, Izumi YOSHIOKA ${ }^{3}$ and Osamu YAMAMOTO ${ }^{1}$

${ }^{1}$ Division of Dermatology, Department of Medicine of Sensory and Motor Organs, Faculty of Medicine, Tottori University, 86 Nishi-cho, Yonagoshi, Tottori 683-8503, ${ }^{2}$ Division of Anatomy, Department of Health Improvement and ${ }^{3}$ Division of Oral Medicine, Department of Oral and Maxillofacial Reconstructive Surgery, Kyushu Dental University, Kokurakita-ku, Kitakyushu, Japan. E-mail: yukoehara@med.tottori-u.ac.jp Accepted Dec 14, 2021; Epub ahead of print Dec 14, 2021

Rhabdomyomatous mesenchymal hamartoma (RMH) is a rare malformation involving the dermis and subcutaneous tissue. It was first described by Hendrick et al. in 1986 as a striated muscle hamartoma (1) and was later termed RMH by Mills in 1989 (2). We report here 5 cases of RMH, review previously reported cases, and propose a new classification of acquired type RMH.

\section{CASE REPORTS}

\section{Clinical histories}

Case 1. A 46-year-old Japanese woman presented with a 3-year history of a normal skin-coloured and asymptomatic plaque on her chin without any trauma. Physical examination revealed a slightly elevated plaque, approximately $20 \times 20 \mathrm{~mm}$ in size, in the centre of the chin. Case 2. A 63-year-old Japanese woman was referred to Tottori University Hospital for evaluation of a 1-month history of a normal skin-coloured plaque on her chin. Physical examination revealed a slightly elevated plaque, approximately $30 \times 25 \mathrm{~mm}$ in size, with dotted areas, in the centre of the chin. Case 3. A 40-year-old Japanese woman was referred to our clinic for evaluation of a 6-month history of a normal skin-coloured plaque on her chin. Physical examination revealed a slightly elevated plaque, approximately $40 \times 35 \mathrm{~mm}$ in size, with a dimple area, on the left side of the chin. Case 4. A 57-year-old Japanese woman was referred to our clinic for evaluation of a 6-month history of a normal skin-coloured plaque with an irregular surface. Physical examination revealed a slightly elevated plaque, approximately $40 \times 25 \mathrm{~mm}$ in size, in the centre of the chin. Case 5. A 52-yearold Japanese woman was referred to our clinic for evaluation of a 6-month history of a normal skin-coloured plaque on her chin. Physical examination revealed a slightly elevated plaque, approximately $20 \times 15 \mathrm{~mm}$ in size, with a dimple area, on the right side of the chin (Fig. 1A).

All of the patients were female (mean age 50 years, age range 39-56 years) and the plaque in all of the cases was on the chin. This study was carried out in accordance with the Declaration of Helsinki and was approved by the ethics committee of Tottori University Faculty of Medicine, Japan (number 18A078).

\section{Histopathological findings (microscopic findings)}

Skin biopsies of the plaques were performed in all 5 cases. Mature bundles of skeletal muscle fibres with typical cross-striation were randomly observed histopathologically in the dermis and subcutaneous tissue. The bundles were running vertically from the subcutaneous tissue to the subepidermal area (Fig. 1B and C). Histochemically, the muscular bundles were recognized as dark-red fascicular structures with Azan stain (Fig. 1D). All cases showed almost identical histopathological findings. Final diagnoses of RMH were made on the basis of those findings.

In addition, we analysed 5 cases of RMH and the marginal normal tissue from the 15 past surgical specimens of the chin in our department. The distance from skin surface to the top of the RMH muscle fibre was approximately $0.5 \pm 0.13 \mathrm{~mm}$. On the other hand, that of normal tissue was approximately $1.14 \pm 0.28$ $\mathrm{mm}$ below the skin surface (not shown).

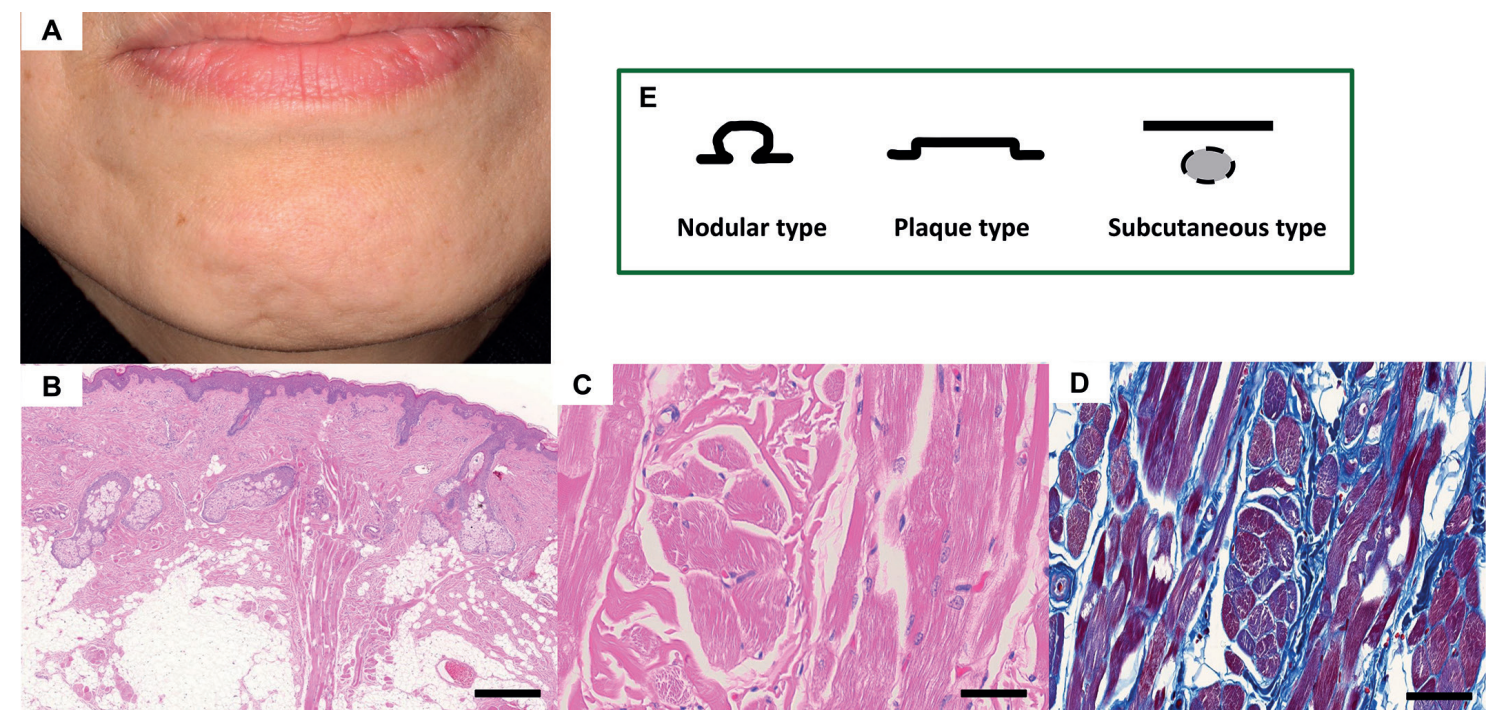

Fig. 1. (A) A skin-coloured plaque on the right side of the chin with a dimple area in case 5 . (B) Mature bundles of skeletal muscle fibres were seen in the dermis and subcutaneous tissue in case 3 (bar: $500 \mu \mathrm{m}$; haematoxylin and eosin (H\&E) staining). (C) The skeletal muscle fibres had cross striation in case 3 (bar: $50 \mu \mathrm{m}$; H\&E staining). (D) The muscle of the bundle was seen as dark red (bar: $100 \mu \mathrm{m}$; Azan staining). (E) Classification of rhabdomyomatous mesenchymal hamartoma (RMH) (nodular type, plaque type and subcutaneous nodule type). 
Table I. Number and frequency of previously reported cases of rhabdomyomatous mesenchymal hamartoma

\begin{tabular}{|c|c|c|}
\hline & \multicolumn{2}{|l|}{ Age of onset } \\
\hline & Congenital type ( $<18$ years old) & Acquired type $\geq 18$ years old) \\
\hline Patients, $n(\%)$ & $65(80)$ & $16(20)$ \\
\hline Sex (male:female), $n$ & $33: 29^{a}$ & $7: 9$ \\
\hline Location, $n(\%)$ & $\begin{array}{l}\text { Head and neck: } 43(66) \text {, oral cavity: } 8(12.5) \text {, trunk: } 8(12.5) \text {, } \\
\text { perianal region: } 6(9)\end{array}$ & Head and neck: 15 (93.7), chin: 7 (43.8), extremities: 1 (6.3) \\
\hline Clinical appearance, $n(\%)$ & $\begin{array}{l}\text { Nodular: } 48(74) \text {, subcutaneous: } 12(18.5), \text { plaque: } 4(6) \text {, } \\
\text { unknown: } 1(1.5)\end{array}$ & Plaque: 7 (43.8), nodular: (6 (37.5), subcutaneous: 3 (18.7) \\
\hline Histopathological feature, $n(\%)$ & Hamartomatous proliferation: $28 / 35$ (80), details unknown: 30 & Hamartomatous proliferation: $3 / 9(33)$, details unknown: 7 \\
\hline
\end{tabular}

aThree unknown.

\section{DISCUSSION}

RMH has been reported under several different names, including striated muscle hamartoma, hamartoma of cutaneous adnexa and mesenchyme, or congenital midline hamartoma. Histopathologically, RMH is characterized by a disordered array of striated muscle bundles and several tissues of mesenchymal origin, such as adipose tissue and vessels in some cases (3). Conversely, in the normal region of the chin, the mentalis muscle is located near the subcutaneous tissue and is directly interfacing with the dermis (4). In summary, the presence of striated muscle tissue just below the epidermis is characteristic for RMH.

The disease commonly presents as a polypoid pedunculated lesion, a dome-shaped sessile nodule, or a subcutaneous mass. It usually develops in newborns/children, but some cases have been reported in adults. It tends to occur in areas containing superficial skeletal muscle, such as the nose or chin, followed by the periorbital region and the anterior neck. The aetiology of RMH is unclear. Aberrant migration of embryonic mesenchymal progenitor cells or abnormal development of mesoderm-derived somite cell populations may be related to the cause of RMH (5). Some RMHs developed in association with spinal dysraphism. These facts suggest that incomplete separation of the neural tube from the overlying ectoderm during embryogenesis disturbs normal mesodermal differentiation and proliferation (6).

As far as we know, to date there have been more than 80 reported cases of RMH including the current cases (7-9). We reviewed those cases according to age of onset and defined them as congenital type (occurring at less than 18 years of age) or acquired type (occurring at 18 years of age or older). We then classified them into 3 clinical types (nodular type, plaque type, and subcutaneous type) (Fig. 1E). Analysis showed that the ratio of patients less than 18 years of age (congenital RMH) was $80 \%$. In this group, $66 \%$ of the tumours were located on the head or neck. In addition, the most common clinical type was nodular type (74\%). Among cases of the acquired type, $93.7 \%$ of the cases developed on the head and neck (with $43.8 \%$ of the cases on the chin). Notably, $43.8 \%$ of the case of acquired RMH were plaque type (Table I).

Although the pathogenesis of RMH has not been elucidated, this study found that the clinical features of congenital and acquired types were different. It is pos- sible that the congenital type is caused by hamartomatous proliferation of not only striated muscles, but also adipose tissues, vessels and nerves. On the other hand, histopathologically, the acquired plaque type showed no proliferation of other mesenchymal components and showed an increased amount of only skeletal muscle fibres. In addition, rather than increasing the density of striated muscles, the striated muscles extended just below the epidermis. The current study indicates that the pathogenesis of congenital RMH may be different from that of acquired plaque type RMH. In 65 previously reported congenital cases of RMH, 35 cases had detailed histopathological descriptions. Among those cases, 28 cases $(80 \%)$ clearly showed hamartomatous hyperplasia. In 16 previously reported acquired cases, detailed histopathology was described in 4 cases of nodular type and 5 cases of plaque type. Three $(75 \%)$ of the 4 cases of nodular type clearly showed hamartomatous proliferation similar to that of congenital RMH. However, all of the 5 plaque type cases exhibited only striated muscle hyperplasia rather than hamartomatous proliferation (Table I) (7-9). We assumed that acquired plaque type was caused by elongation hyperplasia of striated muscles, different from traditional RMH proposed by Hendrick et al.

This study revealed that RMH frequently had different clinical appearances in congenital and acquired cases. We emphasize that the possibility of acquired plaque type RMH should be considered in the differential diagnosis of an adult-onset tumour on the chin.

The authors have no conflicts of interest to declare.

\section{REFERENCES}

1. Hendrick SJ, Sanchez RL, Blackwell SJ, Raimer SS. Striated muscle hamartoma: description of two cases. Pediatr Dermatol 1986; 3: 153-157.

2. Mills AE. Rhabdomyomatous mesenchymal hamartoma of skin. Am J Dermatopathol 1989; 11: 58-63.

3. Read RW, Burnstine M, Rowland JM, Zamir E, Rao NA. Rhabdomyomatous mesenchymal hamartoma of the eyelid: report of a case and literature review. Ophthalmology 2001; 108: 798-804.

4. Beer GM, Manestar M, Mihic-Probst D. The causes of the nasolabial crease: a histomorphological study. Clin Anat 2013; 26: 196-203.

5. Ashfaq R, Timmons CF. Rhabdomyomatous mesenchymal hamartoma of skin. Pediatr Pathol 1992; 12: 731-735.

6. Takeyama J, Hayashi T, Sanada T, Shimanuki Y, Saito M, Shirane R. Rhabdomyomatous mesenchymal hamartoma 
associated with nasofrontal meningocele and dermoid cyst. J Cutan Pathol 2005; 32: 310-313.

7. Kim H, Chung JH, Sung HM, Kim S. Rhabdomyomatous mesenchymal hamartoma presenting as a midline mass on a chin. Arch Craniofac Surg 2017; 18: 292-295.

8. McKinnon EL, Rand AJ, Selim MA, Fuchs HE, Buckley AF,
Cummings TJ. Rhabdomyomatous mesenchymal hamartoma presenting as a sacral skin tag in two neonates with spinal dysraphism. J Cutan Pathol 2015; 42: 774-778.

9. Scrivener Y, Petiau P, Rodier-Bruant C, Cribier B, Heid E, Grosshans E. Perianal striated muscle hamartoma associated with hemangioma. Pediatr Dermatol 1998; 15: 274-276. 